\title{
Gênese Instrumental Pessoal e Conceitos Matemáticos em Processo de Criação com o GeoGebra
}

\author{
Márcia Notare - PPGEMat/UFRGS - marcia.notare@ gmail.com \\ Marcus Vinicius de Azevedo Basso - PPGEMat/UFRGS - PPGIE - mbasso@ufrgs.br
}

Resumo Neste trabalho discute-se o papel do processo de gênese instrumental pessoal na criação de atividades que exploram o conceito de funções em suas diferentes representações. Discute-se também o papel da compreensão de noções matemáticas na elaboração de atividades sobre funções com a utilização de software de geometria dinâmica. De caráter qualitativo, neste estudo são analisadas atividades matemáticas criadas e resolvidas por professores em processo de formação com base na teoria da gênese instrumental de Rabardel. Os resultados apontam para a imbricação entre a gênese instrumental pessoal e a compreensão de conceitos matemáticos no processo de criação utilizando o software GeoGebra.

Palavras-chave: gênese instrumental pessoal, conceituação matemática, geometria dinâmica

\section{Personal Instrumental Genesis and Mathematical Concepts in the Creation Process with GeoGebra}

\begin{abstract}
This paper discusses the role of the process of personal instrumental genesis in the elaboration of activities that explore the concept of functions in their different representations. We also discuss the role of the understanding of mathematical notions in the elaboration of activities on functions with the use of software of dynamic geometry. Of qualitative character, in this study are analyzed mathematical activities created and solved by teachers in process of formation based on the theory of the instrumental genesis of Rabardel. The results point to the imbrication between personal instrumental genesis and the understanding of mathematical concepts in the creation process using GeoGebra software.
\end{abstract}

Keywords: personal instrumental genesis, mathematical conceptualization, dynamic geometry

\section{Introdução}

A utilização do software de matemática dinâmica GeoGebra em salas de aula da Educação Básica encontra-se em uma fase de ascensão, na qual professores de Matemática estão aventurando-se em incorporar esse recurso em seus planejamentos didáticos (Basso e Notare, 2015; Abar e Alencar, 2013).

Entretanto, verifica-se que algumas propostas de utilização do GeoGebra para a aprendizagem de Matemática ainda encontram-se em fase inicial, nas quais o recurso não é explorado com todo seu potencial. Esse fato está ligado ao processo de apropriação tecnológica do professor de Matemática. Para entender esse processo, buscamos suporte teórico na abordagem instrumental de Rabardel (1995), que estuda o movimento de transformação de um artefato em instrumento. Nesse artigo discutimos o papel do processo de gênese instrumental pessoal na criação de atividades com o GeoGebra que exploram o conceito de funções, para evidenciar que atividades com potencial dinâmico ${ }^{1}$ para o estudo de funções resultam da imbricação entre a gênese instrumental pessoal e a compreensão de conceitos matemáticos.

\footnotetext{
${ }^{1}$ No contexto desse artigo, o termo dinâmico refere-se ao comportamento de objetos que podem ser movimentados na tela do computador a partir de relações geométricas ou algébricas.
} 


\section{A gênese instrumental}

A gênese instrumental envolve dois conceitos chaves - artefato e instrumento, e o processo de transformação do primeiro no segundo. Para Rabardel (1995), artefato é o objeto material ou simbólico em si, ou parte de um artefato mais complexo, enquanto que instrumento é definido como uma entidade mista, composta pelo artefato mais os esquemas de utilização elaborados pelo sujeito. A elaboração e evolução do instrumento é um processo longo e complexo, o qual Rabardel (1995) denominou de gênese instrumental. Dessa forma, o processo de gênese instrumental é determinado pela distinção que se estabelece entre artefato e instrumento e pelo processo de transformação progressiva do artefato em instrumento, que nasce da relação entre sujeito e objeto.

Existem dois processos envolvidos na gênese instrumental, denominados instrumentalização e instrumentação. A instrumentalização consiste na emergência e evolução dos componentes do artefato, ou seja, consiste em um progressivo reconhecimento das potencialidades e das limitações do artefato por parte do sujeito (sujeito $\rightarrow$ objeto). A instrumentação consiste na emergência e desenvolvimento de esquemas de utilização (objeto $\rightarrow$ sujeito). Os dois processos, ilustrados no esquema da Figura 1, são orientados, respectivamente, do sujeito para o objeto e vice-versa, e constituem partes inseparáveis da gênese instrumental (Notare e Basso, 2017).

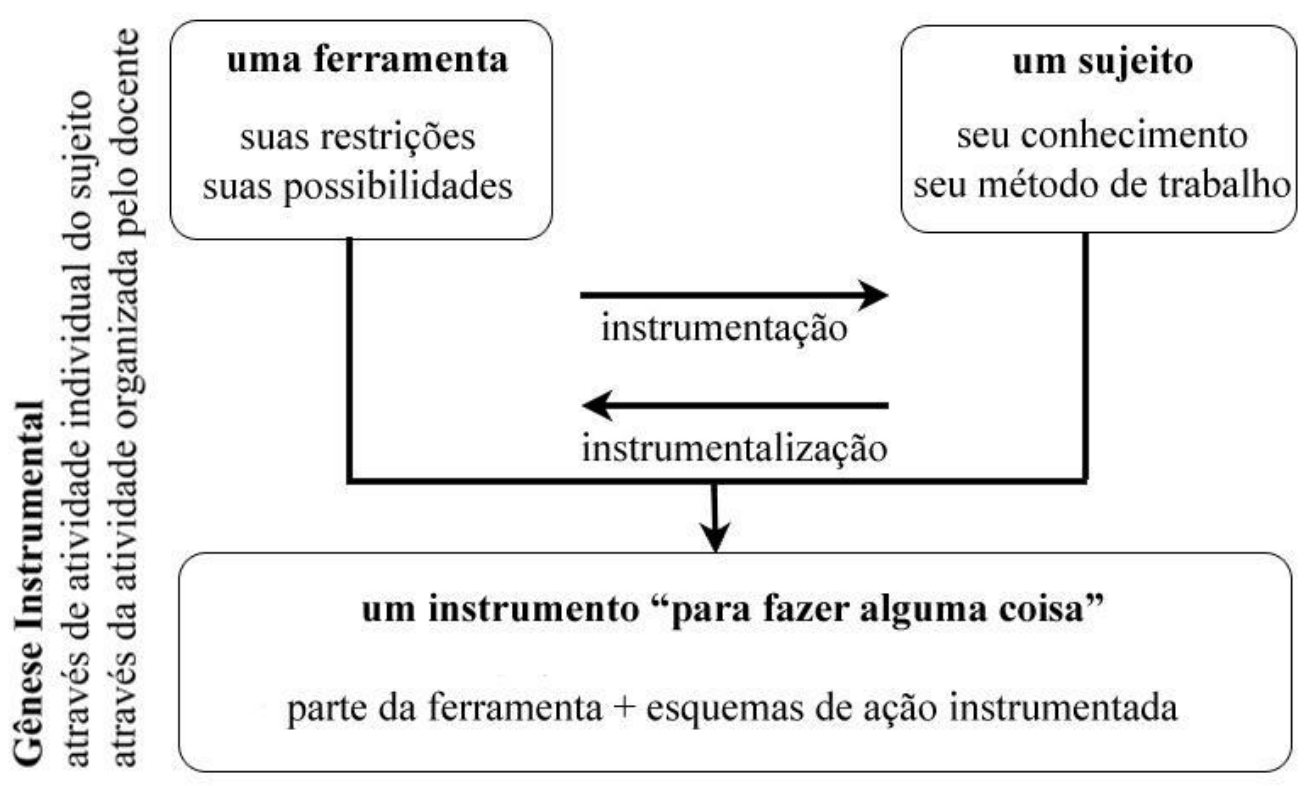

Figura 1 - Esquema ilustrativo do processo de gênese instrumental Fonte: traduzido de Trouche (2004)

No processo de instrumentação o artefato imprime uma marca no sujeito, enquanto que o processo de instrumentalização engloba dois estágios: um estágio de descoberta e seleção de funções relevantes do artefato, e um estágio de personalização e de transformação do artefato. Dessa forma, um instrumento é uma construção individual de cada sujeito, uma vez que a elaboração de esquemas é progressivamente elaborada a partir do uso do artefato em tarefas particulares. Assim, um mesmo artefato transformase em um instrumento diferente para cada sujeito, a partir de esquemas de utilização particulares.

Rabardel (1995) classifica os esquemas de utilização da seguinte forma: (1) esquemas de uso, que estão presentes em ações específicas e diretamente relacionadas com o artefato. Esses esquemas estão relacionados a tarefas secundárias, que são executadas como parte de uma tarefa maior; (2) esquemas de ação instrumentada, que 
englobam esquemas de uso, em uma totalidade, para executar a tarefa maior e mais complexa.

Quando pensamos na gênese instrumental de determinado recurso tecnológico pelo professor de Matemática, verificamos que se trata de um processo complexo, que exige uma apropriação tecnológica, na qual o professor precisa desvendar o artefato, identificando suas potencialidades e também suas limitações. Os processos de exploração e reconhecimento dos componentes do artefato estão imbricados com os conhecimentos matemáticos do professor. Noções matemáticas limitadas implicam na exploração limitada do software e, consequentemente, na elaboração de um instrumento restrito. Por outro lado, conhecimento matemático amplo contribui para que o professor identifique no software ferramentas e componentes que possibilitem construções ricas para a aprendizagem de Matemática. Nesse processo, na medida em que o artefato transforma-se em instrumento, o professor começa a considerar o software com uma ferramenta didática com potencial para a aprendizagem de Matemática.

Porém, segundo Stormowski et al. (2015, p. 7), os cursos de formação de professores de Matemática para o uso de tecnologia, em sua maioria, vêm concentrando esforços no desenvolvimento de esquemas de uso, ou seja, em "ensinar ao professor como o software funciona". No entanto, entendemos que a formação deveria ser centrada no desenvolvimento de esquemas de ação instrumentada, para que o professor seja capaz de realizar tarefas com o artefato. Na seção a seguir discutimos essas relações com o software GeoGebra.

\section{Gênese instrumental e o potencial de atividades}

No caso específico do uso do GeoGebra, foco deste artigo, entendemos que o mesmo se torna um instrumento potente para a aprendizagem de Matemática quando o professor reconhece e explora o dinamismo do software. Explorar e utilizar o recurso de "arrastar" objetos matemáticos com o mouse para observar regularidades nas construções e delas abstrair conhecimentos e relações matemáticas, revelam um processo de elaboração de instrumento mais avançado. $\mathrm{O}$ uso de diferentes representações para um mesmo objeto matemático também é um recurso potente do GeoGebra para a aprendizagem de Matemática. Reconhecer que um mesmo objeto matemático pode ser representado de diferentes formas e provocar a mobilização destas representações de forma simultânea pode levar à construção ampla dos conceitos matemáticos envolvidos. Porém, instigar o trabalho com mais de uma representação para um mesmo objeto matemático exige do professor o domínio amplo desse objeto.

Assim, queremos mostrar que as limitações de uso de uma ferramenta pelo professor de Matemática interferem no processo de pensar a sala de aula com essa ferramenta e, consequentemente, interferem no processo de aprendizagem dos alunos, pois as propostas didáticas com o uso do recurso podem se tornar limitadas, sem tirar o devido proveito do potencial que a ferramenta pode oferecer (Haspekian, 2013).

Como suporte para nossas colocações, analisamos exemplos de atividades com o GeoGebra que exploram o conceito de funções. Classificaremos essas atividades em três níveis: 1, 2 e 3, os quais refletem o progresso no uso do recurso digital. Defendemos aqui o aspecto dinâmico que pode ser empregado no estudo de funções, cujo foco está no estudo do comportamento variacional das funções, no entendimento de suas relações e gráficos de forma qualitativa e simultânea, e não na simples correspondência de pontos estática, que não leva ao entendimento global do comportamento da função.

Uma das atividades que encontramos facilmente em materiais que propõe o estudo de funções com o GeoGebra consiste na "digitação", no campo de entrada do GeoGebra, de algumas relações algébricas com alterações de parâmetros para 
"observar" o que ocorre com os gráficos. Por exemplo, uma atividade que consiste em digitar as relações $y=x^{2}, y=x^{2}+1, y=x^{2}-1$, para que os alunos observem $\mathrm{o}$ comportamento de cada gráfico está longe de explorar o potencial dinâmico que o software proporciona, bem como as possibilidades de trabalho com múltiplas representações. Entendemos que se trata de uma atividade que, apesar de atraente e diferente das aulas com giz e quadro-negro, encontra-se no nível 1 e não provoca reflexões matemáticas que levem à compreensão qualitativa da função quadrática (Figura 2). Nesse caso, o GeoGebra apresenta-se como um instrumento ainda limitado para o professor. $O$ processo de instrumentalização precisa de elaboração de novos esquemas de utilização, que ampliem o uso do artefato, para que o professor explore mais os recursos dinâmicos do GeoGebra e, como resposta, o GeoGebra imprima nesse professor marcas que permitam personalizar seu uso, desenvolvendo esquemas de ação instrumentada.

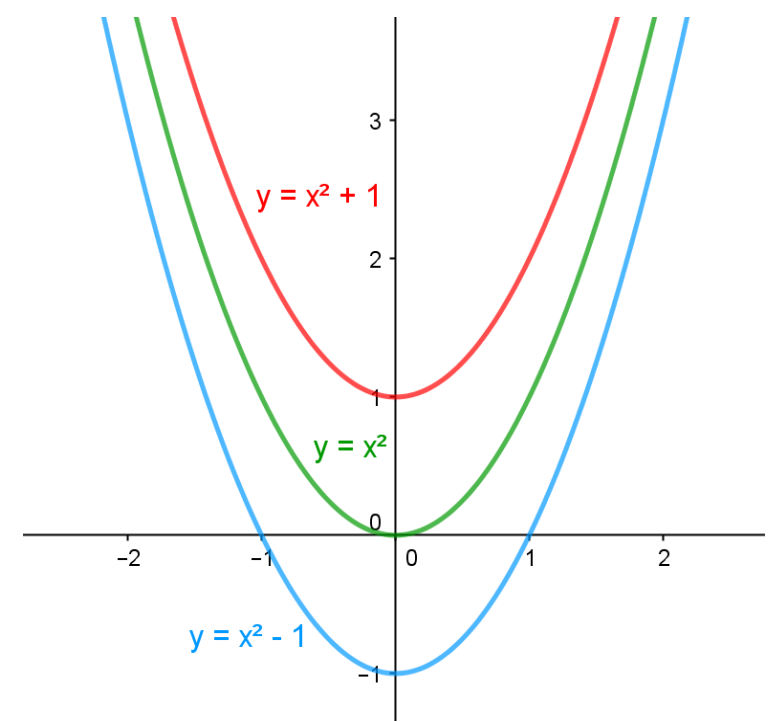

Figura 2 - Exemplo de atividade de nível 1

Fonte: acervo dos autores

Algumas propostas de atividades avançam no sentido de explorar um pouco mais o potencial dinâmico do GeoGebra, acrescentando o uso de controles deslizantes para impor um caráter mais dinâmico e contínuo na atividade. São atividades do tipo "mova o controle deslizante e observe o que acontece", representativa do que denominamos de nível 2. A Figura 3 ilustra uma atividade dessa natureza, na qual explora a relação $y=a x^{2}+b x+c$, em que $a, b$ e $c$ são parâmetros controlados pelos controles deslizantes. Ao movimentar os controles deslizantes, o aluno pode visualizar a alteração do comportamento gráfico, com o objetivo de entender o papel de cada parâmetro algébrico no comportamento gráfico da função. Revela-se aqui um professor cujo instrumento GeoGebra está em uma fase intermediária, na qual recursos relevantes de dinamismo estão sendo desvendados e permitindo a elaboração de esquemas de utilização mais amplos. 


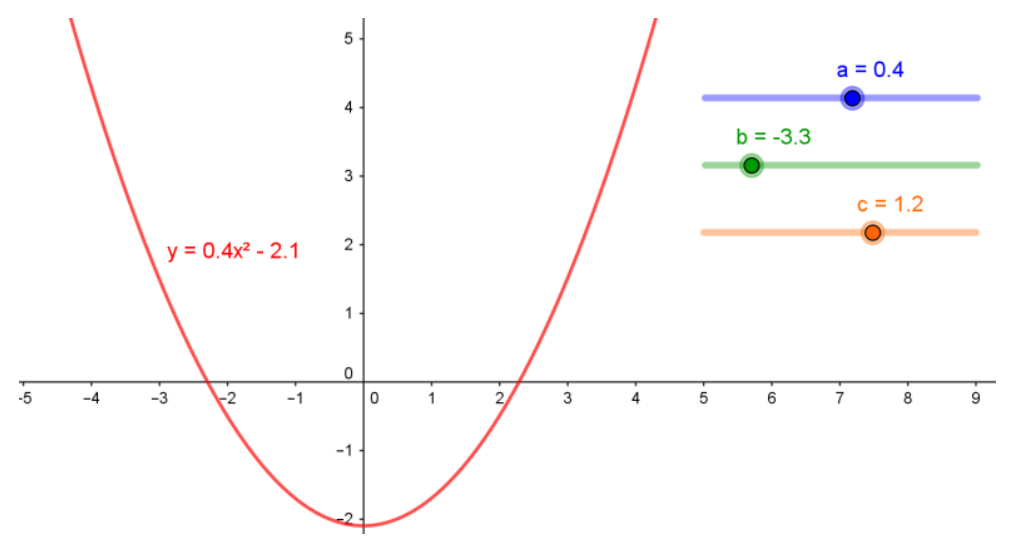

Figura 3 - Exemplo de atividade de nível 2

Fonte: acervo dos autores

Um terceiro exemplo que trazemos para discussão avança para a elaboração de um instrumento mais complexo, cuja utilização conduz a uma atividade com maior potencial (nível 3) para compreensão do conceito variacional de funções e suas múltiplas representações. A atividade consiste em, a partir de uma situação geométrica dinâmica, identificar variáveis e relações funcionais entre elas. A partir da exploração da construção geométrica, é proposto ao aluno que: (1) identifique as variáveis envolvidas na situação geométrica; (2) analise a construção da representação geométrica das variáveis; (3) analise a construção da representação gráfica da relação entre as variáveis; (4) identifique a expressão algébrica representativa dessa função.

A Figura 4 ilustra um exemplo de atividade dessa natureza. Nessa atividade, apresenta-se aos alunos a seguinte situação geométrica: um quadrado de lado constante $k$ e um ponto livre, denominado MOVER, sobre um dos lados do quadrado. A partir desse ponto, constrói-se um novo quadrado, de lado $l$ variável. A pergunta que se faz é: Qual o comportamento variacional da área do quadrado de lado $l$, quando movemos o ponto MOVER? O aluno deve fazer uma exploração qualitativa da situação, movimentando o ponto e fazendo conjecturas sobre o comportamento da área do quadrado: Aumenta? Diminui? Como ocorre essa variação qualitativamente? Quais os possíveis valores para $l$ ? Após uma exploração qualitativa da situação, o aluno deve identificar a relação algébrica que a descreve.

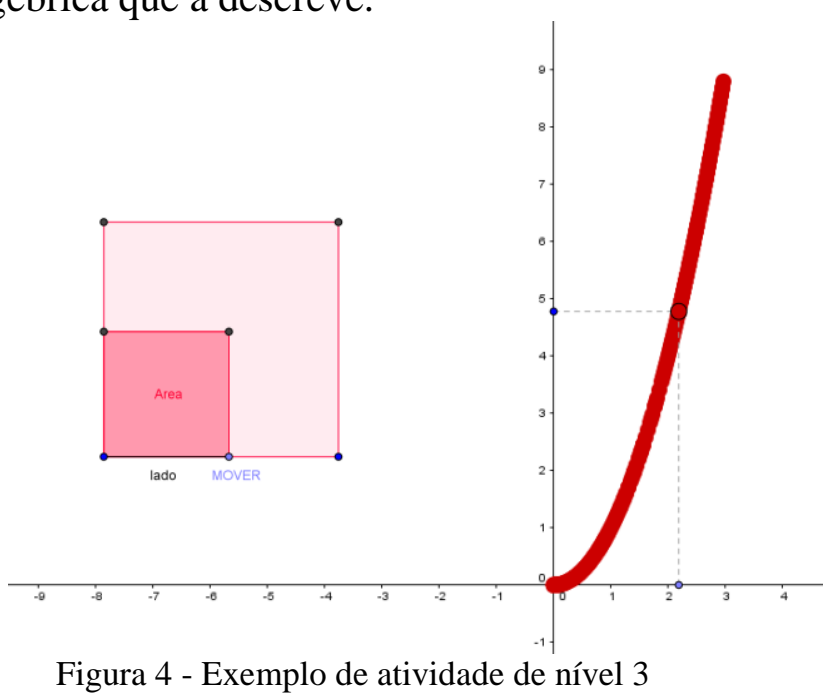

Fonte: acervo de dados de pesquisa dos autores

Aqui, entram em jogo novas exigências cognitivas, que impõem a utilização e construção de conhecimento matemático mais amplo, que mobiliza o trabalho com três representações distintas para o mesmo objeto matemático: representação geométrica, 
representação gráfica e representação algébrica. O aluno sai da posição de "observar o que acontece" para "construir o que acontece". Do ponto de vista do professor que cria a atividade, é preciso que novos esquemas de utilização do GeoGebra sejam elaborados para articular simultaneamente a construção geométrica estável, o ponto livre que se move sobre o lado do quadrado e impacta dinâmica e simultaneamente no ponto que se move no sistema de coordenadas cartesianas. Percebe-se que se trata de uma situação mais complexa, na qual esquemas de uso específicos não são suficientes, exigindo a presença de esquemas de ação instrumentada. Destaca-se também que o domínio do conhecimento matemático que permite determinar a relação algébrica é crucial nessa atividade. $\mathrm{O}$ conhecimento matemático do professor deve extrapolar os limites estáticos dos livros didáticos, que reduz o estudo de funções à construção de tabelas que estabelecem pares ordenados e marcação destes pontos no sistema de coordenadas cartesianas.

Assim, como o instrumento é uma construção individual, cada professor de Matemática utiliza o GeoGebra como instrumento de maneira diferente, a partir de seus esquemas próprios de uso do artefato, de sua experiência pessoal e de suas noções de Matemática. Assim, a elaboração e a utilização de esquemas de utilização do artefato estão relacionadas com a experiência de cada professor e, de acordo com esta experiência, estes esquemas vão sendo modificados, ampliados e/ou integrados aos esquemas já existentes.

$\mathrm{Na}$ seção a seguir analisamos a imbricação entre a gênese instrumental pessoal do GeoGebra e o uso e compreensão de conceitos matemáticos na elaboração de atividades por professores de Matemática, mestrandos do Programa de Pós-graduação em Ensino de Matemática da UFRGS.

\section{Sobre o método e a descrição da atividade}

No contexto de uma disciplina com ênfase no uso de tecnologia digitais para Educação Matemática, a partir da temática Geometria e Funções no GeoGebra, foi proposta uma atividade envolvendo relações entre situações geométricas e funções. Para isso os estudantes/professores deveriam, ao longo do processo de apropriação dos recursos do GeoGebra nesse contexto, vivenciar três etapas: (I) exploração no GeoGebra de uma construção pronta, na qual a ênfase foi a exploração da situação geométrica para a construção e compreensão da matemática que a descreve; (II) construção no GeoGebra de situações geométricas propostas pelos docentes, na qual a ênfase foi a exploração e identificação dos recursos do software; e (III) criação de uma nova situação, na qual os estudantes deveriam preocupar-se simultaneamente com a criação de uma situação com potencial para a construção de conhecimento matemático e rica em termos de recursos do GeoGebra utilizados.

Em todas as etapas, os estudantes tiveram como fio condutor a seguinte proposta: (1) identificar as variáveis envolvidas na situação geométrica; (2) analisar a construção da representação geométrica das variáveis; (3) analisar a construção da representação gráfica da relação entre as variáveis; (4) identificar a expressão algébrica representativa da função. A primeira etapa tratou da situação descrita na seção anterior e ilustrada na Figura 4. A segunda etapa sugeriu a construção de duas situações geométricas, ilustradas nas Figuras 5 e 6. 


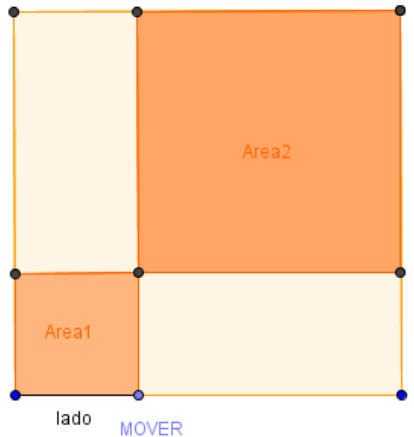

Figura 5 - área de quadrados

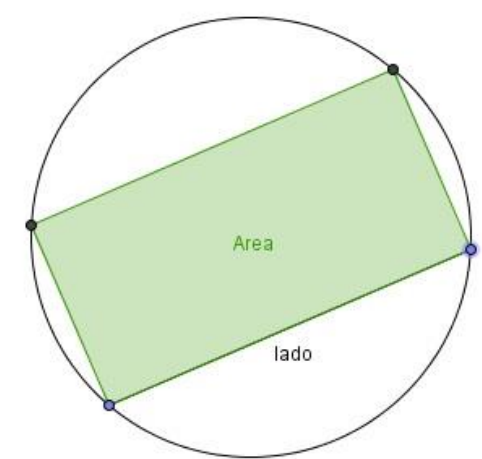

Figura 6 - área de retângulo

Fonte: acervo de dados de pesquisa dos autores

Para a situação da Figura 5, deveria ser tomada como variável dependente a soma das áreas dos quadrados destacados. Para a situação da Figura 6, a variável dependente a ser considerada, deveria ser a área do retângulo inscrito na circunferência.

Finalmente, na etapa III, propunha-se uma construção livre, no qual os quatro passos elencados fossem contemplados. Serão essas construções livres o objeto das análises que apresentaremos. Para isso foram selecionados dois casos exemplares de construções nos quais se procura demonstrar os processos de gênese instrumental pessoal, a compreensão de conceitos matemáticos e a relação entre esses dois processos na criação de uma atividade com o GeoGebra.

Optou-se pelo estudo de casos múltiplos, na linha do que preconiza Ponte (2006, p.2), "Um estudo de caso visa conhecer uma entidade bem definida como uma pessoa, uma instituição, um curso, uma disciplina, um sistema educativo, uma política ou qualquer outra unidade social.". No presente estudo, essa escolha vai ao encontro do argumento de Ponte (2006, p. 3) quanto ao uso de estudos de casos na investigação sobre a formação e o conhecimento de matemática do professor. Assim, a seleção de dois casos exemplares se justifica e atende ao interesse do estudo por destacar os processos de gênese instrumental pessoal, a compreensão de conceitos de matemática e a imbricação entre esses processos.

A análise do processo de criação dos estudantes levou em consideração, simultaneamente, o processo de gênese instrumental pessoal e o conhecimento matemático empregado, refletindo nos seguintes aspectos: (1) o estudante estabelece um plano de ação global na criação da atividade, no qual considera uma situação geométrica dinâmica interessante, em que o estabelecimento das variáveis independente e dependente resultem em uma relação algébrica desafiadora; (2) as construções realizadas são gerais ou representam um caso particular e, neste caso, o professor toma consciência da particularidade de sua construção; (3) qual o nível de conhecimento matemático que se pode identificar nas criações apresentadas; (4) o trabalho com as múltiplas representações, na qual os objetos matemáticos construídos estão em relação de dependência.

O primeiro caso que analisamos refere-se à criação do aluno Alfa, cuja situação geométrica está ilustrada na Figura 7. A atividade criada consiste em analisar a variação da área do triângulo retângulo ACD (variável dependente) inscrito na circunferência de diâmetro $\mathrm{AD}$ (constante), na medida em que se move o ponto $\mathrm{C}$ e se faz variar a medida do cateto $\mathrm{CD}$ (variável independente). Analisando o arquivo de construção de Alfa, verificamos que se trata de uma construção geral e estável, que explora as representações geométrica e gráfica simultaneamente. 


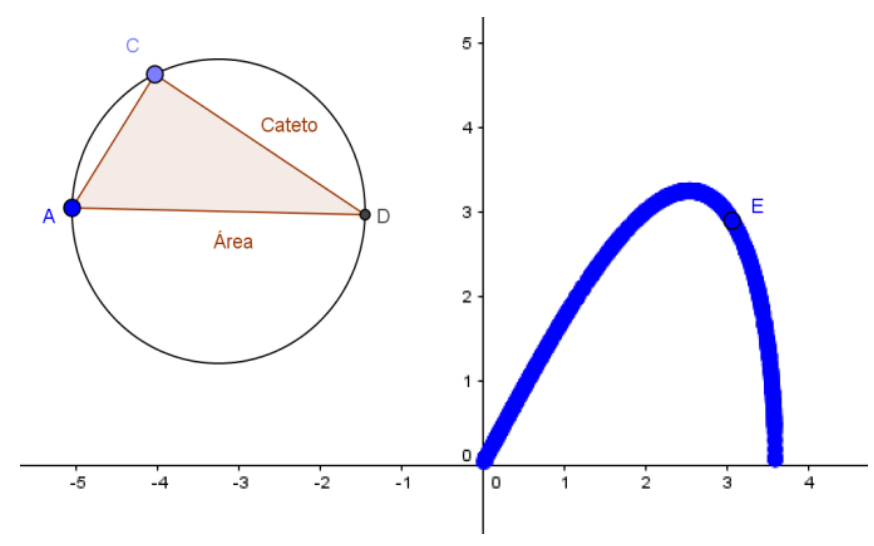

Figura 7 - variação da área do triângulo retângulo Fonte: acervo de dados de pesquisa dos autores

Alfa apresenta também um arquivo de texto, no qual realizada uma análise qualitativa do comportamento variacional da situação geométrica (extrato 1) e identifica a relação algébrica corretamente (extrato 2).

\begin{tabular}{|c|c|}
\hline $\begin{array}{l}\text { Quando } \mathrm{C}=\mathrm{D} \text {, a área é nula e a } \\
\text { coordenada fica situada em }(0,0) \text {. A } \\
\text { área cresce à medida que } \mathrm{C} \text { se afasta } \\
\text { de } \mathrm{D} \text { até atingir seu maior valor } \\
\text { quando } \mathrm{AC}=\mathrm{DC} \text {. Após decresce até } \\
\text { zerar novamente quando } \mathrm{C}=\mathrm{A} \text {. Temos } \\
\text { uma curva côncava para baixo e } \\
\text { assimétrica em relação a reta } \\
\text { Cateto }=r \sqrt{2} \text {. }\end{array}$ & $\begin{array}{l}\text { Isolamos o outro cateto y usando o Teorema de Pitágoras: } \\
(2 \mathrm{r})^{2}=\text { Cateto }^{2}+\mathrm{y}^{2}, \mathrm{y}=\sqrt{4 \mathrm{r}^{2}-\text { Cateto }^{2}} \\
\text { Sabendo que a área é } \frac{\mathrm{b} \cdot \mathrm{h}}{2}: \\
\mathrm{A}(\text { Cateto })=\frac{\left[\text { Cateto } \cdot \sqrt{4 \mathrm{r}^{2}-\text { Cateto }^{2}}\right]}{2} \text {, com } r \in[0,+\infty]\end{array}$ \\
\hline
\end{tabular}

Extrato 1 - comportamento variacional

Extrato 2 - relação algébrica

Percebe-se, ao analisar a criação de Alfa, que o mesmo estabeleceu um plano de ação global, ao escolher uma situação geométrica que representa uma relação algébrica interessante. Os conhecimentos matemáticos de Alfa permitiram que o mesmo criasse uma atividade com potencial para a construção de conceitos de funções envolvendo radical, seu comportamento variacional, sua relação algébrica e respectivo gráfico, além dos conceitos geométricos de triângulo retângulo (catetos e hipotenusa), circunferência (diâmetro e raio), teorema de Pitágoras.

A situação é desafiadora, no sentido de provocar o aluno a compreender qualitativamente como a área do triângulo varia. Ao visualizar o gráfico que é construído dinamicamente pelo movimento do ponto $\mathrm{C}$, fica a provocação sobre qual relação algébrica a descreve. Identificamos apenas um equívoco na determinação do domínio da função: ora, se a variável independente é o cateto $\mathrm{CD}$, então este varia dentro do intervalo $(0,2 \mathrm{r})$. Do ponto de vista da gênese instrumental pessoal de Alfa, temos uma construção estável e geral, o apelo ao dinamismo que o GeoGebra como instrumento para a compreensão da variabilidade das funções proporciona e o uso de diferentes representações para o mesmo objeto matemático. Realçamos que, para a criação avançasse mais no uso das múltiplas representações, e "comprovasse" a relação algébrica determinada por Alfa, o mesmo poderia incluí-la no campo de entrada do GeoGebra, para visualizar os gráficos dinâmico e deduzido sendo confrontados (Figura 10). Contudo, percebe-se que os esquemas de utilização do GeoGebra de Alfa permitem a criação de atividades com potencial dinâmico para o entendimento das noções matemáticas e seu conhecimento matemático potencializa esse processo de apropriação do GeoGebra, conduzindo à criação de uma atividade interessante. 


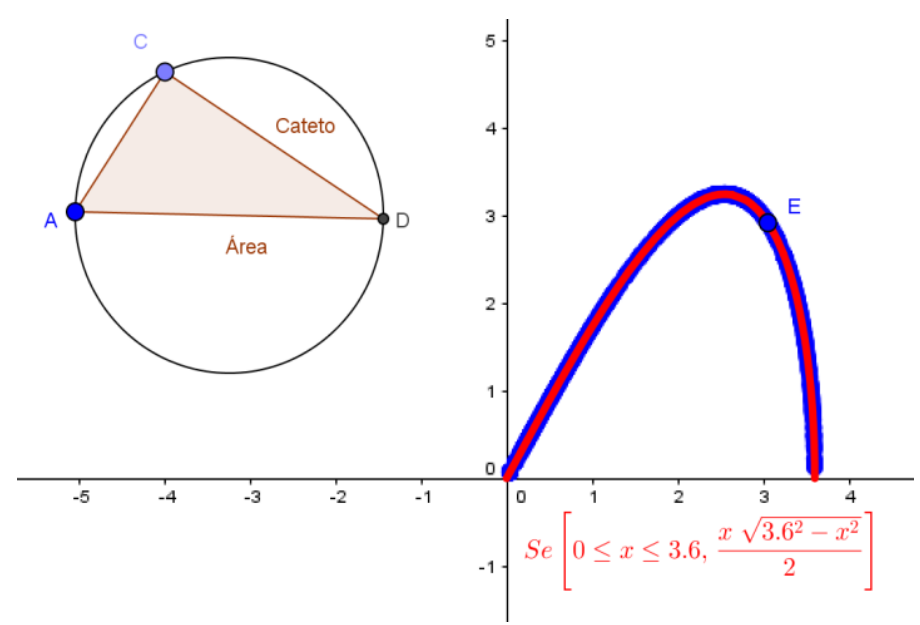

Figura 10 - comportamento variacional, relação algébrica e representação gráfica Fonte: acervo de dados de pesquisa dos autores

$\mathrm{O}$ segundo caso analisado refere-se à criação de Beta (Figura 11). A atividade propõe a análise da variação da área do quadrado inscrito na circunferência de raio $r$ (variável dependente) em função da variação do diâmetro da circunferência (variável independente). De imediato, observamos que a nomenclatura da variável independente não foi adequada, uma vez que usualmente utiliza-se $r$ para referenciar o raio da circunferência. Analisando a proposta de Beta, identificamos uma construção geral e estável, que revela esquemas de utilização que permitem reconhecer o GeoGebra como um instrumento didático para a aprendizagem da Matemática. Beta representa sua situação nas representações geométrica, gráfica e algébrica, o que evidencia apropriação do recurso tecnológico. Contudo, as relações matemáticas que emergem da situação proposta são um tanto elementares, pois a situação geométrica dinâmica criada não desafia o aluno a explorar e conjecturar sobre as possibilidades de variação da área do quadrado. As relações estabelecidas entre as variáveis são imediatas: ao aumentar o diâmetro, a área do quadrado aumenta. Temos aqui um caso em que a gênese instrumental pessoal revela bons esquemas de utilização do GeoGebra, mas os conhecimentos matemáticos não conduziram à criação de uma situação interessante e desafiadora. A formação matemática do professor é um fator crucial para que o uso das tecnologias digitais faça diferença na aprendizagem dos estudantes.

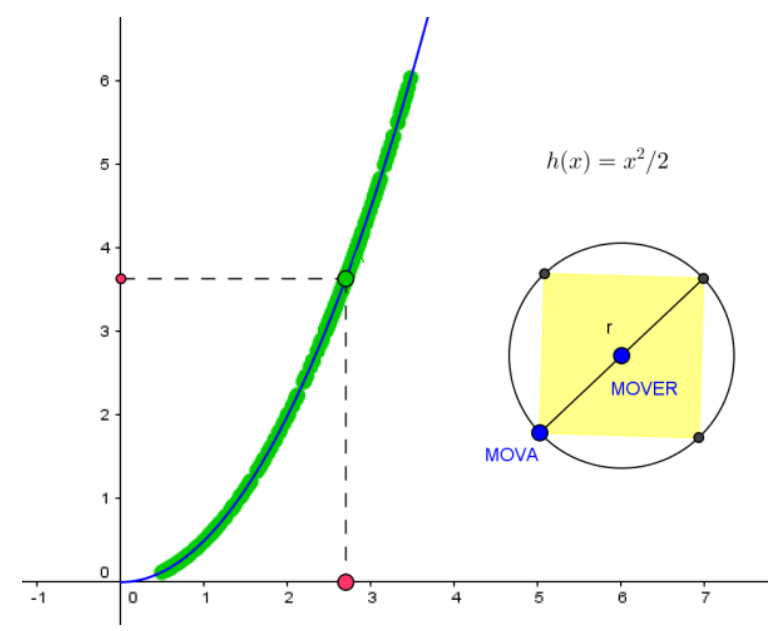

Figura 11 - comportamento variacional, relação algébrica e representação gráfica Fonte: acervo de dados de pesquisa dos autores

O processo de criação de atividades no GeoGebra com potencial para a aprendizagem de Matemática apoia-se, de forma simultânea, na gênese instrumental 
pessoal e no conhecimento matemático do professor. Uma formação para o uso das tecnologias digitais precisa considerar ambos os aspectos.

\section{Considerações finais}

A introdução das tecnologias digitais no processo de formação de professores de Matemática, seja em etapa inicial, continuada ou na pós-graduação, tem se intensificado nos últimos anos. Embora necessária, essa introdução representa apenas uma das etapas necessárias para a inclusão das tecnologias digitais nos processos de ensino e de aprendizagem em ambiente escolar. A inexistência dessa etapa na formação docente pode levar à situação do professor utilizar tecnologias digitais na sala de aula, revelando uma gênese instrumental profissional, sem, no entanto, ter passado por um amplo e consistente processo de gênese instrumental pessoal. Numa situação como a descrita, o reconhecimento e uso das potencialidades do software ficam limitados. Como pensar e criar atividades interessantes e relevantes do ponto de vista da aprendizagem em Matemática sem se apropriar do software?

Em cursos de formação de professores de Matemática, se argumenta a respeito da importância de o professor dominar os conceitos e fundamentos da Matemática para melhor ensinar e propor situações de aprendizagem para os estudantes. Tal argumento não perdeu sua validade. No entanto, ao inserir a componente das tecnologias digitais nesse processo de formação, surgem novas perspectivas. Na perspectiva de Rabardel, consideramos que os processos de gênese instrumental pessoal e profissional estão imbricados com a primeira implicando na segunda. Acrescenta-se que, assim como a apropriação da tecnologia é fundamental, seu uso profissional, via elaboração de bons instrumentos, são dependentes do conhecimento matemático do professor.

\section{Referencias}

ABAR, C. A. A. P; ALENCAR, S. V. (2013). A Gênese Instrumental na Interação com o GeoGebra: uma proposta para a formação continuada de professores de Matemática. Boletim de Educação Matemática. Rio Claro, SP, v.27, n. 46, agosto, p.349-365.

BASSO, M. V. A.; NOTARE, M. R. (2015). Pensar-com Tecnologias Digitais de Matemática Dinâmica. Revista Novas Tecnologias na Educação, v. 13, n. 2, p. 1-10.

HASPEKIAN, M. (2013). Enseigner avec un tableur: quelles genèses instrumentales? In: Les technologies numériques pour l'énseignement. Toulouse: OCTARÈS Editions.

NOTARE, M. R., BASSO, M.V.A. (2017). Gênese Instrumental do GeoGebra na Formação de Professores. Zetetiké, Campinas, SP, v.25, maio/ago.2017, p.324-344.

PONTE, J. P. (2006). Estudos de Caso em Educação Matemática. Boletim de Educação Matemática, Rio Claro, v.19, n.25, p.105-132.

RABARDEL, P. (1995). Les hommes et les technologies: une approche cognitive des instruments contemporains. Paris: Armand Colin.

TROUCHE, L. (2004). Managing the complexity human/machine interactions in computerized learning environment: guiding student's command process through instrumental orchestrations. International Journal of Computers for Mathematical Learning, Netherlands, n.9, p.281-307.

STORMOWSKI, V.; GRAVINA, M.A.; \& LIMA, J.V. (2015). Formação de professores de matemática para o uso efetivo de tecnologias em sala de aula. Revista Novas Tecnologias na Educação, v.13, n.2, p 1-10. 